\title{
Critical Fujita exponents to a class of non-Newtonian filtration equations with fast diffusion
}

\author{
Mingjun Zhou' ${ }^{1}$, Huilai Li', Wei Guo ${ }^{2^{*}}$ (I) and Xu Zhou ${ }^{3}$
}

\section{"Correspondence:}

guoweijilin@163.com

¿School of Mathematics and

Statistics, Beihua University, Jilin,

132013, China

Full list of author information is

available at the end of the article

\section{照 Springer}

\begin{abstract}
We consider the Cauchy problem to a class of fast-diffusion non-Newtonian filtration equations. Besides the usual degeneracy in the fast-diffusion non-Newtonian filtration, the equation is degenerate or singular at infinity, depending on the sign of the parameter related to the coefficient of diffusion. Fujita type theorems are established and the critical Fujita exponent is determined. Specially, we also prove that the nontrivial solution blows up in a finite time on the critical situation.
\end{abstract}

MSC: $35 \mathrm{~K} 55 ; 35 \mathrm{~B} 33$

Keywords: critical Fujita exponent; degeneracy; singularity; non-Newtonian filtration equation; fast diffusion

\section{Introduction}

The purpose of this paper is to investigate the critical Fujita exponent for the following initial value problem:

$$
\begin{aligned}
& (|x|+1)^{\mu_{1}} \frac{\partial u}{\partial t}=\operatorname{div}\left(|\nabla u|^{q-1} \nabla u\right)+(|x|+1)^{\mu_{2}} u^{p}, \quad x \in \mathbb{R}^{n}, t>0, \\
& u(x, 0)=u_{0}(x), \quad x \in \mathbb{R}^{n},
\end{aligned}
$$

where $p>1,0<q<1, \max \{-n,(n-1) / q-(n+1)\}<\mu_{1} \leq \mu_{2}<p \mu_{1}+(p-1) n$, and $0 \leq u_{0} \in$ $C_{0}\left(\mathbb{R}^{n}\right)$.

The study of critical exponents began in 1966 by Fujita in [1], where it was proved for the initial value problem of

$$
\frac{\partial u}{\partial t}=\Delta u+u^{p}, \quad x \in \mathbb{R}^{n}, t>0
$$

that the problem admits no nontrivial nonnegative global solution if $1<p<p_{c}=1+2 / n$, whereas if $p>p_{c}$, it admits both global (with small data) and non-global (with large initial data) solutions. Later, in 1981, Weissler [2] proved that the critical case $p=p_{c}$ is still a blow-up case.

In Fujita's work, the new phenomenon of nonlinear parabolic equations was discovered. From then on, there has been a lot of work on the critical Fujita exponents for various non-

(c) 2016 Zhou et al. This article is distributed under the terms of the Creative Commons Attribution 4.0 International License (http://creativecommons.org/licenses/by/4.0/), which permits unrestricted use, distribution, and reproduction in any medium, provided you give appropriate credit to the original author(s) and the source, provide a link to the Creative Commons license, and indicate if changes were made. 
linear evolution equations and systems (see, e.g., the survey papers $[3,4]$ and the references therein, and also [5-15]). Among those, the Fujita type theorems for the slow-diffusion non-Newtonian filtration equation

$$
\frac{\partial u}{\partial t}=\operatorname{div}\left(|\nabla u|^{q-1} \nabla u\right)+u^{p}, \quad x \in \mathbb{R}^{n}, t>0
$$

was investigated by Galaktionov in $[16,17]$, where $p, q>1$. He proved that $p_{c}=q+(q+$ $1) / n$ by blow-up subsolutions and global supersolutions. Recently, the same problem for an interesting variant of (3) is studied by the authors [13]. The non-Newtonian filtration equations with fast diffusion were considered by Qi and Wang in [18], where the critical Fujita exponent was determined for the Cauchy problem of the equation

$$
\frac{\partial u}{\partial t}=\operatorname{div}\left(|\nabla u|^{q-1} \nabla u\right)+|x|^{\sigma} u^{p}, \quad x \in \mathbb{R}^{n}, t>0
$$

with $p>1,(n-1) /(n+1)<q<1$, and $\sigma>n(1-q)-q-1$. It is shown that $p_{c}=q+(q+1+\sigma) / n$ by energy functions. Obviously, they did not cover the portion $0<q \leq(n-1) /(n+1)$ of the fast-diffusion range.

In the present paper, we study the problem (1), (2) and formulate the critical Fujita exponent as

$$
p_{c}=q+\left(q+1+\mu_{2}\right) /\left(n+\mu_{1}\right)
$$

and the critical situation $p=p_{c}$ is still the blow-up case. The range of $m$ considered in this paper is $0<m<1$, the whole fast-diffusion range of (1). Like the non-Newtonian filtration equation with fast diffusion, (1) is singular at points where $|\nabla u|=0$. In addition, (1) is degenerate at $|x|=+\infty$ for $\mu_{1}>0$ and singular for $\mu_{1}<0$, different from both (3) and (4). Inspired by $[11,18,19]$, to prove the solutions' blow-up, we analyze the interaction between the nonlinear source and nonlinear diffusion via precise estimates through constructing energy functions by use of the normalized principal eigenfunction of $-\Delta$ in the unit ball $B_{1}$ of $\mathbb{R}^{n}$ with homogeneous initial-boundary condition, rather than constructing subsolutions as the author did in [16, 17]. This method for equation (1) and its special case (4) basically depends upon the nonincreasing properties in the spatial variant of solutions, which is trivial with $\mu_{1}=\mu_{2}$, while it may be invalid if $\mu_{1}<\mu_{2}$. For all these reasons, we have to overcome some technical difficulties.

This paper is arranged as follows. Some preliminaries are introduced in Section 2, including the local existence theorem, the comparison principle, and a property of solutions from propagation of disturbances. The Fujita type theorems are established in Section 3. Finally in Section 4, the critical case will be concerned.

\section{Preliminaries}

Throughout this paper, we use $B_{r}$ to indicate the ball in $\mathbb{R}^{n}$ with radius $r$ and center at the origin. The solution considered here is taken in the following sense.

Definition 2.1 We call

$$
0 \leq u \in C\left([0, T) ; L^{\infty}\left(\mathbb{R}^{n}\right)\right) \cap L_{\mathrm{loc}}^{q+1}\left(0, T ; W_{\mathrm{loc}}^{1, q+1}\left(\mathbb{R}^{n}\right)\right)
$$


a solution to the problem (1), (2) in $(0, T)$ with $0<T \leq+\infty$ if

$$
\int_{0}^{T} \int_{\mathbb{R}^{n}}\left((|x|+1)^{\mu_{1}} u \frac{\partial \phi}{\partial t}-|\nabla u|^{q-1} \nabla u \cdot \nabla \phi+(|x|+1)^{\mu_{2}} u^{p} \phi\right) d x d t=0
$$

holds for any $\phi \in C_{0}^{\infty}\left(\mathbb{R}^{n} \times(0, T)\right)$ and

$$
\lim _{t \rightarrow 0^{+}} \int_{\mathbb{R}^{n}} u(x, t) \zeta(x) d x=\int_{\mathbb{R}^{n}} u_{0}(x) \zeta(x) d x
$$

for any $\zeta \in C_{0}^{\infty}\left(\mathbb{R}^{n}\right)$.

Like the non-Newtonian filtration equation, it is not hard to prove the well-posedness to the problem (1), (2), one can see, e.g., [20].

Next, we will prove the following proposition on a property of solutions from propagation of disturbances.

Proposition 2.1 Assume that $u$ is a solution to the problem (1), (2) with $0 \leq u_{0} \in C_{0}\left(\mathbb{R}^{n}\right)$ nontrivial, then $u\left(0, t_{0}\right)>0$ for some $t_{0}>0$.

Proof That $u_{0}$ is nontrivial shows that there exists $0 \neq x_{0} \in \mathbb{R}^{n}$ and $\kappa, \rho>0$ such that

$$
u_{0}(x) \geq \kappa\left(1-\left(\frac{\left|x-x_{0}\right|^{q+1}}{\rho^{q+1}}\right)^{1 / q}\right)_{+}^{2}, \quad x \in \mathbb{R}^{n}
$$

where $s_{+}=\max \{s, 0\}$. Let

$$
\begin{aligned}
& \Phi(x, t)=\frac{\kappa \rho^{(q+1) \xi}}{R^{\xi}(t)}\left(1-\left(\frac{\left|x-x_{0}\right|^{q+1}}{R(t)}\right)^{1 / q}\right)_{+}^{2}, \quad x \in \mathbb{R}^{n}, t>0, \\
& D=\left\{(x, t) \in \mathbb{R}^{n+1} \times \mathbb{R}_{+}:\left|x-x_{0}\right|<2\left|x_{0}\right|,\left|x-x_{0}\right|^{q+1}<R(t), 0<t<\frac{\kappa^{1-q} \rho^{q+1}}{\xi}\right\},
\end{aligned}
$$

with $R(t)=\kappa^{q-1} t+\rho^{q+1}$, and $\xi>1$ independent of $\kappa$ and $\rho$ to be chosen later.

Denote

$$
\|z\|=\frac{\left|x-x_{0}\right|^{q+1}}{R(t)}, \quad H=1-\|z\|^{1 / q} .
$$

A direct calculation within $D$ shows

$$
\begin{aligned}
& \frac{\partial \Phi}{\partial t}=-\frac{\xi \kappa^{q} \rho^{(q+1) \xi}}{R^{\xi+1}(t)} H^{2}+\frac{2}{q} \frac{\kappa^{q} \rho^{(q+1) \xi}}{R^{\xi+1}(t)} H\|z\|^{1 / q}, \\
& \operatorname{div}\left(|\nabla \Phi|^{q-1} \nabla \Phi\right)=-\left(\frac{2(q+1)}{q}\right)^{q} \frac{1}{R(t)}\left(\frac{\kappa \rho^{(q+1) \xi}}{R^{\xi}(t)} H\right)^{q}\left(n-(q+1) \frac{\|z\|^{1 / q}}{H}\right) .
\end{aligned}
$$

Setting

$$
\mathscr{L}[\Phi]=\frac{R^{\xi+1}(t)}{\kappa^{q} \rho^{(q+1) \xi} H}\left((|x|+1)^{\mu_{1}} \frac{\partial \Phi}{\partial t}-\operatorname{div}\left(|\nabla \Phi|^{q-1} \nabla \Phi\right)\right), \quad(x, t) \in D,
$$


then

$$
\begin{aligned}
\mathscr{L}[\Phi]= & -(|x|+1)^{\mu_{1}} \xi H+\frac{2}{q}(|x|+1)^{\mu_{1}}\|z\|^{1 / q} \\
& +\left(\frac{2(q+1)}{q}\right)^{q}\left(\frac{\rho^{(q+1) \xi}}{R^{\xi}(t)} H\right)^{q-1}\left(n-(q+1) \frac{\|z\|^{1 / q}}{H}\right) .
\end{aligned}
$$

Divide $D$ into two sets

$$
D^{(1)}=\{(x, t) \in D: H<\delta\} \quad \text { and } \quad D^{(2)}=\{(x, t) \in D: H \geq \delta\}
$$

with $\delta>0$ satisfying

$$
\delta^{q-1}\left((q+1)\left(\frac{1}{\delta}-1\right)-n\right) \geq \frac{2}{q} \Lambda_{1}
$$

where

$$
\Lambda_{1}= \begin{cases}\left(3\left|x_{0}\right|+1\right)^{\mu_{1}}, & \mu_{1} \geq 0, \\ 1, & \mu_{1}<0 .\end{cases}
$$

Then in $D^{(1)}$,

$$
\begin{aligned}
\mathscr{L}[\Phi] & \leq \frac{2}{q} \Lambda_{1}+\left(\frac{2(q+1)}{q}\right)^{q} \delta^{q-1}\left(n-(q+1)\left(\frac{1}{\delta}-1\right)\right) \\
& \leq \frac{2}{q} \Lambda_{1}+\delta^{q-1}\left(n+q+1-\frac{q+1}{\delta}\right) \\
& \leq 0 .
\end{aligned}
$$

For the chosen $\delta>0$, we have in $D^{(2)}$

$$
\mathscr{L}[\Phi] \leq-\Lambda_{2} \xi \delta+\frac{2}{q} \Lambda_{1}+n\left(\frac{2(q+1)}{q}\right)^{q}\left(\frac{\rho^{(q+1) \xi}}{R^{\xi}(t)} \delta\right)^{q-1},
$$

where

$$
\Lambda_{2}= \begin{cases}1, & \mu_{1} \geq 0 \\ \left(3\left|x_{0}\right|+1\right)^{\mu_{1},}, & \mu_{1}<0 .\end{cases}
$$

Due to

$$
\left(\frac{\rho^{(q+1) \xi}}{R^{\xi}(t)} \delta\right)^{q-1} \leq\left(1+\frac{1}{\xi}\right)^{\xi(1-q)} \delta^{q-1} \leq\left(\frac{\mathrm{e}}{\delta}\right)^{1-q}, \quad(x, t) \in D
$$

we know

$$
\mathscr{L}[\Phi] \leq-\Lambda_{2} \xi \delta+\frac{2}{q} \Lambda_{1}+n\left(\frac{2(q+1)}{q}\right)^{m}\left(\frac{\mathrm{e}}{\delta}\right)^{1-q}, \quad(x, t) \in D .
$$


So for fixed $\delta>0$ satisfying (5) and $\xi>1$ satisfying

$$
\Lambda_{2} \xi \delta \geq \frac{2}{q} \Lambda_{1}+n\left(\frac{2(q+1)}{q}\right)^{q}\left(\frac{\mathrm{e}}{\delta}\right)^{1-q}
$$

we obtain

$$
(|x|+1)^{\mu_{1}} \frac{\partial \Phi}{\partial t}-\operatorname{div}\left(|\nabla \Phi|^{q-1} \nabla \Phi\right) \leq 0, \quad x \in \mathbb{R}^{n}, 0<t<\frac{\kappa^{1-q} \rho^{q+1}}{\xi}
$$

Clearly, the constant $\xi>1$ is independent of $\kappa$ and $\rho$. The comparison principle implies

$$
u(x, t) \geq \Phi(x, t), \quad(x, t) \in D
$$

In particular,

$$
u\left(x, t_{1}\right)>0, \quad x \in \Gamma_{1}
$$

with $t_{1}=\frac{\kappa^{1-q} \rho^{q+1}}{\xi}$ and

$$
\Gamma_{1}=\left\{x \in \mathbb{R}^{n}:\left|x-x_{0}\right|<2\left|x_{0}\right|,\left|x-x_{0}\right|^{q+1}<\frac{\xi+1}{\xi} \rho^{q+1}\right\} .
$$

If $0 \in \Gamma_{1}$, the proof is complete. Otherwise,

$$
u\left(x, t_{1}\right) \geq \Phi\left(x, t_{1}\right)=\kappa_{1}\left(1-\left(\frac{\left|x-x_{0}\right|^{q+1}}{\rho_{1}^{q+1}}\right)^{1 / q}\right)_{+}^{2}, \quad x \in \mathbb{R}^{n}
$$

where

$$
\kappa_{1}=\kappa\left(\frac{\xi}{\xi+1}\right)^{\xi}, \quad \rho_{1}=\rho\left(\frac{\xi+1}{\xi}\right)^{1 /(q+1)} .
$$

From the above argument, we have

$$
u(x, t) \geq \Phi_{1}(x, t), \quad(x, t) \in D_{1},
$$

where

$$
\begin{aligned}
& \Phi_{1}(x, t)=\frac{\kappa_{1} \rho_{1}^{(q+1) \xi}}{R_{1}^{\xi}(t)}\left(1-\left(\frac{\left|x-x_{0}\right|^{q+1}}{R_{1}(t)}\right)^{1 / q}\right)_{+}^{2}, \quad x \in \mathbb{R}^{n}, t>t_{1}, \\
& D_{1}=\left\{(x, t) \in \mathbb{R}^{n} \times \mathbb{R}_{+}:\left|x-x_{0}\right|<2\left|x_{0}\right|,\left|x-x_{0}\right|^{q+1}<R_{1}(t), t_{1}<t<t_{1}+\frac{\kappa_{1}^{1-q} \rho_{1}^{q+1}}{\xi}\right\},
\end{aligned}
$$

with $R_{1}(t)=\kappa_{1}^{q-1}\left(t-t_{1}\right)+\rho_{1}^{q+1}$. In particular,

$$
u\left(x, t_{2}\right)>0, \quad x \in \Gamma_{2},
$$


with $t_{2}=t_{1}+\frac{\kappa_{1}^{1-q} \rho_{1}^{q+1}}{\xi}$ and

$$
\Gamma_{2}=\left\{x \in \mathbb{R}^{n}:\left|x-x_{0}\right|<2\left|x_{0}\right|,\left|x-x_{0}\right|^{q+1}<\frac{\xi+1}{\xi} \rho_{1}^{q+1}\right\} .
$$

If $0 \in \Gamma_{2}$, the proof is complete. Otherwise, repeat the above procedure. We get the conclusion in finite steps.

\section{Fujita type theorems}

Let us establish the Fujita type theorems.

Definition 3.1 We call $u$ the blow-up solution to equation (1) if there exists some $0<T_{*}<$ $+\infty$ such that

$$
\lim _{t \rightarrow T_{*}^{-}}\|u(\cdot, t)\|_{L^{\infty}\left(\mathbb{R}^{n}\right)}=\lim _{t \rightarrow T_{*}^{-}} \sup _{x \in \mathbb{R}^{n}} u(x, t)=+\infty
$$

Theorem 3.1 Assume that $1<p<p_{c}=q+\left(q+1+\mu_{2}\right) /\left(n+\mu_{1}\right)$ and $0 \leq u_{0} \in C_{0}\left(\mathbb{R}^{n}\right)$ is nontrivial. Then the problem (1), (2) admits a blow-up solution.

Proof Due to Proposition 2.1, we may assume $u_{0}(0)>0$. By the comparison principle, we only need to prove the conclusion for radial and nonincreasing $u_{0}(x)$, i.e.,

$$
u_{0}(x)=h_{0}(|x|), \quad x \in \mathbb{R}^{n},
$$

where $h_{0} \in C_{0}^{1}([0,+\infty))$ satisfies $h_{0}^{\prime}(0)=0$ and $h_{0}^{\prime}(r) \leq 0$ for $r>0$. With such initial data, the solution $u$ is also radial, namely

$$
u(x, t)=h(|x|, t), \quad x \in \mathbb{R}^{n}, t \geq 0 .
$$

If $\mu_{1}=\mu_{2}$, it is easy to know that $u$ is also nonincreasing by a standard regularization argument and the maximum principle. However, this method is invalid if $\mu_{1}<\mu_{2}$. In the following discussion, we will first of all consider a nonincreasing $u$, namely $h(r, t)$ is nonincreasing with respect to $r \in[0,+\infty)$ for any $t \geq 0$, and we treat the general case finally.

Let

$$
\psi(x)= \begin{cases}1, & 0 \leq|x| \leq 1 \\ f(|x|-1), & 1<|x|<2, \\ 0, & |x| \geq 2\end{cases}
$$

where $f$ is the principal eigenfunction of $-\Delta$ in the unit ball $B_{1}$ of $\mathbb{R}^{n}$ with homogeneous initial-boundary condition, normalized by $\|f\|_{L^{\infty}\left(B_{1}\right)}=1$. For $l>1$, define

$$
\psi_{l}(x)=\psi(x / l), \quad x \in \mathbb{R}^{n}
$$

Then

$$
\left|\nabla \psi_{l}\right| \leq \frac{M_{0}}{l}, \quad\left|\Delta \psi_{l}\right| \leq \frac{M_{0}}{l^{2}}, \quad \frac{\left|\Delta \psi_{l}\right|}{\psi_{l}} \leq \frac{M_{0}}{l^{2}}, \quad x \in B_{2 l} \backslash B_{l},
$$


Chou et al. Boundary Value Problems (2016) 2016:146

Page 7 of 16

with $M_{0}>0$ independent of $l$. Set

$$
\eta_{l}(t)=\int_{\mathbb{R}^{n}}(|x|+1)^{\mu_{1}} u \psi_{l} d x, \quad t \geq 0
$$

Definition 2.1 gives

$$
\frac{d \eta_{l}}{d t}=-\int_{B_{2 l}}|\nabla u|^{q-1} \nabla u \cdot \nabla \psi_{l} d x+\int_{\mathbb{R}^{n}}(|x|+1)^{\mu_{2}} u^{p} \psi_{l} d x .
$$

For radial and nonincreasing $u(x, t)$, one has

$$
\begin{aligned}
\int_{B_{2 l}}|\nabla u|^{q-1} \nabla u \cdot \nabla \psi_{l} d x & =\int_{B_{2 l}}|\nabla u|^{q}\left|\nabla \psi_{l}\right| d x \\
& \leq\left(\int_{B_{2 l}}|\nabla u| \cdot\left|\nabla \psi_{l}\right| d x\right)^{q}\left(\int_{B_{2 l}}\left|\nabla \psi_{l}\right| d x\right)^{1-q} \\
& =\left(\int_{B_{2 l}} \nabla u \cdot \nabla \psi_{l} d x\right)^{q}\left(\int_{B_{2 l}}\left|\nabla \psi_{l}\right| d x\right)^{1-q} \\
& \leq M_{0} l^{(n-1)(1-q)}\left(\int_{B_{2 l}} \nabla u \cdot \nabla \psi_{l} d x\right)^{q}
\end{aligned}
$$

and

$$
0 \leq \int_{B_{2 l}} \nabla u \cdot \nabla \psi_{l} d x=\int_{\partial B_{2 l}} u \nabla \psi_{l} \cdot v d \sigma-\int_{B_{2 l}} u \Delta \psi_{l} d x \leq-\int_{B_{2 l}} u \Delta \psi_{l} d x,
$$

where $\boldsymbol{v}$ is the unit outer normal to $\partial B_{2 l}$. Hence

$$
\frac{d \eta_{l}}{d t} \geq-M_{0} l^{(1-q)(n-1)}\left|\int_{B_{2 l}} u \Delta \psi_{l} d x\right|^{q}+\int_{\mathbb{R}^{n}}(|x|+1)^{\mu_{2}} u^{p} \psi_{l} d x .
$$

The Hölder inequality yields

$$
\begin{aligned}
\left|\int_{B_{2 l}} u \Delta \psi_{l} d x\right|^{q} \leq & \left(\int_{B_{2 l} \backslash B_{l}} u\left|\Delta \psi_{l}\right| d x\right)^{q} \\
\leq & \left(\int_{B_{2 l} \backslash B_{l}}(|x|+1)^{-\mu_{2} /(p-1)}\left|\Delta \psi_{l}\right|^{p /(p-1)} \psi_{l}^{-1 /(p-1)} d x\right)^{q(p-1) / p} \\
& \cdot\left(\int_{B_{2 l} \backslash B_{l}}(|x|+1)^{\mu_{2}} u^{p} \psi_{l} d x\right)^{q / p} \\
\leq & M_{1} l^{q(n-2)-q\left(n+\mu_{2}\right) / p}\left(\int_{\mathbb{R}^{n}}(|x|+1)^{\mu_{2}} u^{p} \psi_{l} d x\right)^{q / p}
\end{aligned}
$$

with $M_{1}>0$ independent of $l$, which, together with (8), implies

$$
\begin{aligned}
\frac{d \eta_{l}}{d t} \geq & \left(\int_{\mathbb{R}^{n}}(|x|+1)^{\mu_{2}} u^{p} \psi_{l} d x\right)^{q / p} \\
& \cdot\left\{-M_{0} M_{1} l^{n-q-1-q\left(n+\mu_{2}\right) / p}+\left(\int_{\mathbb{R}^{n}}(|x|+1)^{\mu_{2}} u^{p} \psi_{l} d x\right)^{(p-q) / p}\right\} .
\end{aligned}
$$


By the Hölder inequality,

$$
\begin{aligned}
\int_{\mathbb{R}^{n}}(|x|+1)^{\mu_{1}} u \psi_{l} d x \leq & \left(\int_{B_{2 l}}(|x|+1)^{\left(p \mu_{1}-\mu_{2}\right) /(p-1)} \psi_{l} d x\right)^{(p-1) / p} \\
& \cdot\left(\int_{\mathbb{R}^{n}}(|x|+1)^{\mu_{2}} u^{p} \psi_{l} d x\right)^{1 / p} \\
\leq & M_{2} l^{\left(n+\mu_{1}\right)-\left(n+\mu_{2}\right) / p}\left(\int_{\mathbb{R}^{n}}(|x|+1)^{\mu_{2}} u^{p} \psi_{l} d x\right)^{1 / p}
\end{aligned}
$$

and hence

$$
\int_{\mathbb{R}^{n}}(|x|+1)^{\mu_{2}} u^{p} \psi_{l} d x \geq M_{2}^{-p} l^{-p\left(n+\mu_{1}\right)+\left(n+\mu_{2}\right)} \eta_{l}^{p}
$$

with $M_{2}>0$ independent of $l$. Equations (9) and (10) show that

$$
\begin{aligned}
\frac{d \eta_{l}}{d t} \geq & \left(M_{2}^{-p} l^{-p\left(n+\mu_{1}\right)+\left(n+\mu_{2}\right)}\right)^{q / p} \eta_{l}^{q} \\
& \cdot\left\{-M_{0} M_{1} l^{n-q-1-q\left(n+\mu_{2}\right) / p}+M_{2}^{q-p} l^{\left[-p\left(n+\mu_{1}\right)+\left(n+\mu_{2}\right)\right](p-q) / p} \eta_{l}^{p-q}\right\} .
\end{aligned}
$$

We mention that the above discussion holds provided that $p>1$.

If $p<p_{c}=q+\left(q+1+\mu_{2}\right) /\left(n+\mu_{1}\right)$, then

$$
n-q-1-\left(n+\mu_{2}\right) q / p<\left[-p\left(n+\mu_{1}\right)+\left(n+\mu_{2}\right)\right](p-q) / p .
$$

Notice that $\eta_{l}$ is nondecreasing with respect to $l \in(1,+\infty)$ and $\sup \left\{\eta_{l}(0): l \in(1,+\infty)\right\}>0$, and from (11) one shows that, for $l>1$ large enough, there exists a constant $\delta>0$ such that

$$
\begin{aligned}
\frac{d \eta_{l}}{d t} \geq & \left(M_{2}^{-p} l^{-p\left(n+\mu_{1}\right)+\left(n+\mu_{2}\right)}\right)^{q / p} \eta_{l}^{q} \\
& \cdot\left(\frac{1}{2} M_{2}^{q-p} l^{\left[-p\left(n+\mu_{1}\right)+\left(n+\mu_{2}\right)\right](p-q) / p} \eta_{l}^{p-q}\right) \\
\geq & \delta \eta_{l}^{p} .
\end{aligned}
$$

So there exists some $0<T_{*}<+\infty$ such that

$$
\lim _{t \rightarrow T_{*}^{-}} \eta_{l}(t)=+\infty
$$

Due to supp $\psi_{l}=B_{2 l}$, we obtain

$$
\lim _{t \rightarrow T_{*}^{-}}\|u(\cdot, t)\|_{L^{\infty}\left(\mathbb{R}^{n}\right)}=+\infty
$$

Next, for the general case without the assumption that $u(x, t)$ is nonincreasing, define

$$
\underline{u}(x, t)=\min _{0 \leq r \leq|x|} h(r, t), \quad x \in \mathbb{R}^{n}, t \geq 0 .
$$


Then $\underline{u}(x, t)$ is nonincreasing,

$$
0 \leq \underline{u}(x, t) \leq u(x, t), \quad x \in \mathbb{R}^{n}, t \geq 0,
$$

and

$$
\frac{d}{d t} \int_{\mathbb{R}^{n}}(|x|+1)^{\mu_{1}} \underline{u} \psi_{l} d x \geq-\int_{B_{2 l}}|\nabla \underline{u}|^{q-1} \nabla \underline{u} \cdot \nabla \psi_{l} d x+\int_{\mathbb{R}^{n}}(|x|+1)^{\mu_{2}} \underline{u}^{p} \psi_{l} d x .
$$

From the above argument, we get

$$
\lim _{t \rightarrow \tilde{T}_{*}^{-}}\|\underline{u}(\cdot, t)\|_{L^{\infty}\left(\mathbb{R}^{n}\right)}=+\infty
$$

for some $0<\tilde{T}_{*}<+\infty$, and (13) ensures that $u$ is a blow-up solution.

Let us turn to the case $p>p_{c}$. Suppose that

$$
U(x, t)=(t+1)^{-\alpha} V\left((t+1)^{-\beta}(|x|+1)\right)
$$

where

$$
\alpha=\frac{q+1+\mu_{2}}{\mu_{1}(p-q)+\mu_{2}(q-1)+(q+1)(p-1)}, \quad \beta=\frac{p-q}{q+1+\mu_{2}} \alpha,
$$

is a self-similar solution to (1). It is easy to show that $V(r)$ solves

$$
\left(\left|V^{\prime}\right|^{q-1} V^{\prime}\right)^{\prime}+\frac{n-1}{r}\left|V^{\prime}\right|^{q-1} V^{\prime}+\beta r^{\mu_{1}+1} V^{\prime}+\alpha r^{\mu_{1}} V+r^{\mu_{2}} V^{p}=0, \quad r>0
$$

Lemma 3.1 Assume that $p>p_{c}=q+\left(q+1+\mu_{2}\right) /\left(n+\mu_{1}\right)$. Then for $\varepsilon>0$ small enough, the function

$$
V(r)=\varepsilon\left(1+\rho(\varepsilon) r^{\lambda}\right)^{-\gamma}, \quad r>0,
$$

where

$$
\lambda=1+\frac{\mu_{1}+1}{q}, \quad \gamma=\frac{q}{1-q}, \quad \rho(\varepsilon)=\frac{1}{\gamma \lambda} \beta^{1 / q} \varepsilon^{(1-q) / q},
$$

is a supersolution to equation (17), i.e.

$$
\left(\left|V^{\prime}\right|^{q-1} V^{\prime}\right)^{\prime}+\frac{n-1}{r}\left|V^{\prime}\right|^{q-1} V^{\prime}+\beta r^{\mu_{1}+1} V^{\prime}+\alpha r^{\mu_{1}} V+r^{\mu_{2}} V^{p} \leq 0, \quad r>0 .
$$

Proof It is not hard to show that it suffices to verify

$$
\begin{aligned}
& q(\gamma+1) \rho(\varepsilon) \lambda[\varepsilon \gamma \lambda \rho(\varepsilon)]^{q}\left[1+\rho(\varepsilon) r^{\lambda}\right]^{-q(\gamma+1)-1} r^{(q+1)(\lambda-1)} \\
& -q(\lambda-1)[\varepsilon \gamma \lambda \rho(\varepsilon)]^{q}\left[1+\rho(\varepsilon) r^{\lambda}\right]^{-q(\gamma+1)} r^{q(\lambda-1)-1} \\
& -(n-1)[\varepsilon \gamma \lambda \rho(\varepsilon)]^{q}\left[1+\rho(\varepsilon) r^{\lambda}\right]^{-q(\gamma+1)} r^{q(\lambda-1)-1}
\end{aligned}
$$




$$
\begin{aligned}
& -\beta \varepsilon \gamma \lambda \rho(\varepsilon)\left[1+\rho(\varepsilon) r^{\lambda}\right]^{-(\gamma+1)} r^{\mu_{1}+\lambda}+\alpha \varepsilon\left[1+\rho(\varepsilon) r^{\lambda}\right]^{-\gamma} r^{\mu_{1}} \\
& +\varepsilon^{p}\left[1+\rho(\varepsilon) r^{\lambda}\right]^{-p \gamma} r^{\mu_{2}} \leq 0, \quad r>0,
\end{aligned}
$$

namely

$$
\begin{aligned}
& \left\{[\varepsilon \gamma \lambda \rho(\varepsilon)]^{q}-\beta \varepsilon\right\} \gamma \lambda \rho(\varepsilon)\left[1+\rho(\varepsilon) r^{\lambda}\right]^{-(\gamma+1)} r^{\mu_{1}+\lambda} \\
& \quad-\left\{[q(\lambda-1)+n-1] \varepsilon^{-1}[\varepsilon \gamma \lambda \rho(\varepsilon)]^{q}-\alpha-\varepsilon^{p-1}\left[1+\rho(\varepsilon) r^{\lambda}\right]^{-(p-1) \gamma} r^{\mu_{2}-\mu_{1}}\right\} \\
& \quad \cdot \varepsilon\left[1+\rho(\varepsilon) r^{\lambda}\right]^{-\gamma} r^{\mu_{1}} \leq 0, \quad r>0 .
\end{aligned}
$$

From the definition of $[\varepsilon \gamma \lambda \rho(\varepsilon)]^{q}=\beta \varepsilon$, we have

$$
\begin{aligned}
& {[q(\lambda-1)+n-1] \varepsilon^{-1}[\varepsilon \gamma \lambda \rho(\varepsilon)]^{q}-\alpha-\varepsilon^{p-1}\left[1+\rho(\varepsilon) r^{\lambda}\right]^{-(p-1) \gamma} r^{\mu_{2}-\mu_{1}}} \\
& \quad=\left(n+\mu_{1}\right) \beta-\alpha-\varepsilon^{p-1}\left[1+\rho(\varepsilon) r^{\lambda}\right]^{-(p-1) \gamma} r^{\mu_{2}-\mu_{1}} \\
& \quad \geq\left(n+\mu_{1}\right) \beta-\alpha-\varepsilon^{p-1}[\rho(\varepsilon)]^{-(p-1) \gamma} r^{\mu_{2}-\mu_{1}-\lambda(p-1) \gamma} .
\end{aligned}
$$

Due to $p>p_{c}=q+\left(q+1+\mu_{2}\right) /\left(n+\mu_{1}\right)$ and $(n-1) / q-(n+1)<\mu_{1} \leq \mu_{2}$,

$$
\left(n+\mu_{1}\right) \beta>\alpha, \quad \lambda(p-1) \gamma \geq \mu_{2}-\mu_{1} \geq 0 .
$$

Hence,

$$
\left(n+\mu_{1}\right) \beta-\alpha-\varepsilon^{p-1}[\rho(\varepsilon)]^{-(p-1) \gamma} r^{\mu_{2}-\mu_{1}-\lambda(p-1) \gamma}>0, \quad r>0
$$

holds for sufficiently small $\varepsilon>0$, and (18) is obtained.

Theorem 3.2 Assume that $p>p_{c}=q+\left(q+1+\mu_{2}\right) /\left(n+\mu_{1}\right)$. Then the solution to the problem (1), (2) exists globally with small $u_{0}$, or blows up with large $u_{0}$.

Proof Let $V(r)$ be a supersolution to equation (17) in Lemma 3.1. From

$$
V^{\prime}(r)<0, \quad r>0
$$

one can show that $U(x, t)$ given in (15) is a supersolution to equation (1) with $\alpha$ and $\beta$ given in (16). Therefore, the comparison principle implies the problem (1), (2) has a nontrivial global solution with small $u_{0}$.

Let us turn to the case of large $u_{0}$. Denote by the radial $u$ a solution to the problem (1), (2). Temporarily suppose $u$ is nonincreasing. Then (11) holds with $\eta_{l}$ defined by (7). If $u_{0}$ is so large that

$$
M_{0} M_{1} l_{0}^{n-q-1-\left(n+\mu_{2}\right) q / p}<\frac{1}{2} M_{2}^{q-p} l_{0}^{\left[-p\left(n+\mu_{1}\right)+\left(n+\mu_{2}\right)\right](p-q) / p} \eta_{l_{0}}^{p-q}(0)
$$

holds for some $l_{0}>1$, then from (11), we get

$$
\eta_{l_{0}}(t) \geq \eta_{l_{0}}(0), \quad t>0
$$


and

$$
\begin{aligned}
\frac{d \eta_{l_{0}}}{d t} \geq & \left(M_{2}^{-p} l_{0}^{-p\left(n+\mu_{1}\right)+\left(n+\mu_{2}\right)}\right)^{q / p} \eta_{l_{0}}^{q} \\
& \cdot\left(\frac{1}{2} M_{2}^{q-p} l_{0}^{\left[-p\left(n+\mu_{1}\right)+\left(n+\mu_{2}\right)\right](p-q) / p} \eta_{l_{0}}^{p-q}\right) \\
= & \delta_{0} \eta_{l_{0}}^{p}
\end{aligned}
$$

for some $\delta_{0}>0$. Therefore, $u$ is a blow-up solution.

For the general case without the assumption that $u(x, t)$ is nonincreasing, considering a new function just as in the proof of Theorem 3.1, one can also see that $u$ is a blow-up solution.

\section{Critical case}

Now, let us deal with the critical case $p=p_{c}=q+\left(q+1+\mu_{2}\right) /\left(n+\mu_{1}\right)$. Let $\psi_{l}, \eta_{l}, M_{1}, M_{2}$ be defined as in the previous section.

Lemma 4.1 Assume that $p=p_{c}=q+\left(q+1+\mu_{2}\right) /\left(n+\mu_{1}\right)$ and $u$ is a nontrivial, global, radial, and nonincreasing solution to the problem (1), (2). Then

$$
\int_{\mathbb{R}^{n}}(|x|+1)^{\mu_{1}} u(x, t) d x \leq M, \quad t>0
$$

holds for some constant $M>0$ independent of $t$.

Proof $p=p_{c}$ yields

$$
n-q-1-\left(n+\mu_{2}\right) q / p_{c}=\left[-p_{c}\left(n+\mu_{1}\right)+\left(n+\mu_{2}\right)\right]\left(p_{c}-q\right) / p_{c} .
$$

Then, for the global, radial and nonincreasing solution $u$, from (11), we have

$$
M_{2}^{q-p_{c}} \eta_{l}^{p_{c}-q}(t) \leq 2 M_{0} M_{1}, \quad l>1, t>0 .
$$

Otherwise, $u$ blows up in a finite time. Therefore (19) holds for some constant $M>0$ owing to

$$
\lim _{l \rightarrow+\infty} \eta_{l}(t)=\int_{\mathbb{R}^{n}}(|x|+1)^{\mu_{1}} u(x, t) d x .
$$

Lemma 4.2 Assume that $p=p_{c}=q+\left(q+1+\mu_{2}\right) /\left(n+\mu_{1}\right), u$ be a nontrivial, radial, and nonincreasing solution to the problem (1), (2) and $0<\theta<1$. Then

$$
\begin{aligned}
\frac{d \eta_{l}}{d t} \geq & M_{2}^{-q(1-\theta)} l^{\left(n+\mu_{2}\right)-p_{c}\left(n+\mu_{1}\right)} \eta_{l}^{q(1-\theta)} \\
& \cdot\left\{-M_{3}\left(\int_{B_{2 l} \backslash B_{l}}(|x|+1)^{\mu_{1}} u \psi_{l} d x\right)^{q \theta}+M_{2}^{q(1-\theta)-p_{c}} \eta_{l}^{p_{c}-q(1-\theta)}\right\}
\end{aligned}
$$

holds for any $l>1$ with a constant $M_{3}>0$ independent of $l$. 
Proof For any $l>1$, the Hölder inequality yields

$$
\begin{aligned}
\left|\int_{B_{2 l}} u \Delta \psi_{l} d x\right|^{q} & \left(\int_{B_{2 l} \backslash B_{l}} u\left|\Delta \psi_{l}\right| d x\right)^{q} \\
\leq & \left(\int_{B_{2 l} \backslash B_{l}}(|x|+1)^{-\left(\theta p_{c} \mu_{1}+(1-\theta) \mu_{2}\right) /\left[\left(p_{c}-1\right)(1-\theta)\right]}\right. \\
& \left.\cdot\left|\Delta \psi_{l}\right|^{p_{c} /\left[\left(p_{c}-1\right)(1-\theta)\right]} \psi_{l}^{-\left(1-\theta+p_{c} \theta\right) /\left[\left(p_{c}-1\right)(1-\theta)\right]} d x\right)^{q\left(p_{c}-1\right)(1-\theta) / p_{c}} \\
& \cdot\left(\int_{B_{2 l} \backslash B_{l}}(|x|+1)^{\mu_{2}} u^{p_{c}} \psi_{l} d x\right)^{q(1-\theta) / p_{c}}\left(\int_{B_{2 l} \backslash B_{l}}(|x|+1)^{\mu_{1}} u \psi_{l} d x\right)^{q \theta} \\
\leq & M l^{q(n-2)-q\left(n+\mu_{2}\right) / p_{c}+q \theta\left(\mu_{2}-p_{c} \mu_{1}-\left(p_{c}-1\right) n\right) / p_{c}} \\
& \cdot\left(\int_{\mathbb{R}^{n}}(|x|+1)^{\mu_{2}} u^{p_{c}} \psi_{l} d x\right)^{q(1-\theta) / p_{c}}\left(\int_{B_{2 l} \backslash B_{l}}(|x|+1)^{\mu_{1}} u \psi_{l} d x\right)^{q \theta},
\end{aligned}
$$

with $M>0$ a constant independent of $l$, which, together with (10) and (8), implies

$$
\begin{aligned}
\frac{d \eta_{l}}{d t} \geq & \left(\int_{\mathbb{R}^{n}}(|x|+1)^{\mu_{2}} u^{p_{c}} \psi_{l} d x\right)^{q(1-\theta) / p_{c}} \\
& \cdot\left\{-M_{0} M l^{(n-1)(1-q)+q(n-2)-q\left(n+\mu_{2}\right) / p_{c}+q \theta\left[\mu_{2}-p_{c} \mu_{1}-\left(p_{c}-1\right) n\right] / p_{c}}\right. \\
& \left.\cdot\left(\int_{B_{2 l} \backslash B_{l}}(|x|+1)^{\mu_{1}} u \psi_{l} d x\right)^{q \theta}+\left(\int_{\mathbb{R}^{n}}(|x|+1)^{\mu_{2}} u^{p_{c}} \psi_{l} d x\right)^{\left[p_{c}-q(1-\theta)\right] / p_{c}}\right\} \\
\geq & \left.\left(M_{2}^{-p_{c}} l^{-p_{c}\left(n+\mu_{1}\right)+\left(n+\mu_{2}\right)}\right)^{q(1-\theta) / p_{c}} \eta_{l}^{q(1-\theta)}\right\} \\
& \cdot\left\{-M_{0} M l^{(n-1)(1-q)+q(n-2)-q\left(n+\mu_{2}\right) / p_{c}+q \theta\left[\mu_{2}-p_{c} \mu_{1}-\left(p_{c}-1\right) n\right] / p_{c}}\right. \\
& \cdot\left(\int_{B_{2 l} \backslash B_{l}}(|x|+1)^{\mu_{1}} u \psi_{l} d x\right)^{q \theta} \\
+ & \left.M_{2}^{q(1-\theta)-p_{c}} \eta_{l}^{p_{c}-q(1-\theta)} l^{\left[-p_{c}\left(n+\mu_{1}\right)+\left(n+\mu_{2}\right)\right]\left[p_{c}-q(1-\theta)\right] / p_{c}}\right\} .
\end{aligned}
$$

Then (20) holds due to

$$
\begin{aligned}
& (n-1)(1-q)+q(n-2)-q\left(n+\mu_{2}\right) / p_{c}+q \theta\left[\mu_{2}-p_{c} \mu_{1}-\left(p_{c}-1\right) n\right] / p_{c} \\
& \quad=\left[-p_{c}\left(n+\mu_{1}\right)+\left(n+\mu_{2}\right)\right]\left[p_{c}-q(1-\theta)\right] / p_{c} .
\end{aligned}
$$

Lemma 4.3 Assume that $p=p_{c}=q+\left(q+1+\mu_{2}\right) /\left(n+\mu_{1}\right)$, u be a nontrivial, radial and nonincreasing solution to the problem (1), (2). Then

$$
\frac{d \eta_{l}}{d t} \geq-M_{4} l^{\left[p_{c}(n-q-1)-q\left(n+\mu_{2}\right)\right] /\left(p_{c}-q\right)}, \quad l>1
$$

holds for some constant $M_{4}>0$ independent of $l$. 
Proof From (9), we have

$$
\begin{aligned}
\frac{d \eta_{l}}{d t} \geq & \int_{\mathbb{R}^{n}}(|x|+1)^{\mu_{2}} u^{p_{c}} \psi_{l} d x \\
& -M l^{(n-1)(1-q)+q\left[n-2-\left(n+\mu_{2}\right) / p_{c}\right]}\left(\int_{\mathbb{R}^{n}}(|x|+1)^{\mu_{2}} u^{p_{c}} \psi_{l} d x\right)^{q / p_{c}} .
\end{aligned}
$$

Then the Young inequality gives

$$
\begin{aligned}
\frac{d \eta_{l}}{d t} \geq & \int_{\mathbb{R}^{n}}(|x|+1)^{\mu_{2}} u^{p_{c}} \psi_{l} d x-\frac{q}{p_{c}} \int_{\mathbb{R}^{n}}(|x|+1)^{\mu_{2}} u^{p_{c}} \psi_{l} d x \\
& -\frac{p_{c}-q}{p_{c}} M^{p_{c} /\left(p_{c}-q\right)} l^{\left[p_{c}(n-q-1)-q\left(n+\mu_{2}\right)\right] /\left(p_{c}-q\right)} \\
\geq & -M_{4} l^{\left[p_{c}(n-q-1)-q\left(n+\mu_{2}\right)\right] /\left(p_{c}-q\right)} .
\end{aligned}
$$

We are ready to prove the blow-up theorem of Fujita type for the critical case $p=p_{c}=$ $q+\left(q+1+\mu_{2}\right) /\left(n+\mu_{1}\right)$.

Theorem 4.1 Assume that $p=p_{c}=q+\left(q+1+\mu_{2}\right) /\left(n+\mu_{1}\right)$ and $u$ is a solution to the problem (1), (2) with $0 \leq u_{0} \in C_{0}\left(\mathbb{R}^{n}\right)$ nontrivial. Then the problem (1), (2) admits a blowup solution.

Proof Similarly to the proof of Theorem 3.1, at first assume $u_{0}$ is radial and nonincreasing. Then $u$ is radial, given by (6). Denote

$$
\Lambda=\sup _{l>1, t>0} \eta_{l}(t)=\sup _{t>0} \int_{\mathbb{R}^{n}}(|x|+1)^{\mu_{1}} u(x, t) d x .
$$

From (19) and the nontriviality of $u, 0<\Lambda<+\infty$. For any $0<\sigma<\Lambda$, due to (22) and $\eta_{l}$ being nondecreasing with respect to $l \in(1,+\infty)$, there exist $\omega_{0} \geq 0$ and $l_{0}>2$ such that

$$
\eta_{l_{0} / 2}\left(\omega_{0}\right) \geq \Lambda-\sigma
$$

Then it follows from (21) that

$$
\begin{aligned}
& \int_{\mathbb{R}^{n}}(|x|+1)^{\mu_{1}} u(x, s) \psi_{l_{0} / 2}(x) d x \\
& \quad \geq \int_{\mathbb{R}^{n}}|x|^{\mu_{1}} u\left(x, \omega_{0}\right) \psi_{l_{0} / 2}(x) d x-M_{4}\left(l_{0} / 2\right)^{\left[p_{c}(n-q-1)-q\left(n+\mu_{2}\right)\right] /\left(p_{c}-q\right)}\left(s-\omega_{0}\right) \\
& \quad \geq \Lambda-\sigma-M_{4}\left(l_{0} / 2\right)^{\left[p_{c}(n-q-1)-q\left(n+\mu_{2}\right)\right] /\left(p_{c}-q\right)}\left(s-\omega_{0}\right), \quad s \geq \omega_{0} .
\end{aligned}
$$

Thus

$$
\begin{aligned}
& \int_{B_{2 l_{0}} \backslash B_{l_{0}}}(|x|+1)^{\mu_{1}} u(x, s) \psi_{l_{0}}(x) d x \\
& \quad \leq \int_{\mathbb{R}^{n}}(|x|+1)^{\mu_{1}} u(x, s) d x-\int_{\mathbb{R}^{n}}(|x|+1)^{\mu_{1}} u(x, s) \psi_{l_{0} / 2}(x) d x \\
& \quad \leq \sigma+M_{4}\left(l_{0} / 2\right)^{\left[p_{c}(n-q-1)-q\left(n+\mu_{2}\right)\right] /\left(p_{c}-q\right)}\left(s-\omega_{0}\right), \quad s \geq \omega_{0} .
\end{aligned}
$$


Choosing $l=l_{0}$ in (20), we have

$$
\begin{aligned}
& \frac{d \eta_{l_{0}}}{d t} \geq M_{2}^{-q(1-\theta)} l_{0}^{\left(n+\mu_{2}\right)-p_{c}\left(n+\mu_{1}\right)} \eta_{l_{0}}^{q(1-\theta)} \\
& \cdot\left\{-M_{3}\left(\int_{B_{2 l_{0}} \backslash B_{l_{0}}}(|x|+1)^{\mu_{1}} u \psi_{l_{0}} d x\right)^{q \theta}+M_{2}^{q(1-\theta)-p_{c}} \eta_{l_{0}}^{p_{c}-q(1-\theta)}\right\} \\
& \geq M_{2}^{-q(1-\theta)} l_{0}^{\left(n+\mu_{2}\right)-p_{c}\left(n+\mu_{1}\right)} \eta_{l_{0}}^{q(1-\theta)} \\
& \cdot\left\{-M_{3}\left(\sigma+M_{4}\left(l_{0} / 2\right)^{\left[p_{c}(n-q-1)-q\left(n+\mu_{2}\right)\right] /\left(p_{c}-q\right)}\left(s-\omega_{0}\right)\right\}\right)^{q \theta} \\
& \left.+M_{2}^{q(1-\theta)-p_{c}} \eta_{l_{0}}^{p_{c}-q(1-\theta)}\right\}, \quad t>\omega_{0} .
\end{aligned}
$$

Fix $\sigma_{0} \in(0, \Lambda)$ and $M_{5}>0$, independent of $l_{0}$, such that

$$
M_{3}\left(\sigma_{0}+M_{5}\right)^{q \theta} \leq \frac{1}{2} M_{2}^{q(1-\theta)-p_{c}}\left(\Lambda-\sigma_{0}\right)^{p_{c}-q(1-\theta)} .
$$

Then

$$
\frac{d \eta_{l_{0}}}{d t} \geq \frac{1}{2} M_{2}^{-p_{c}} l_{0}^{\left(n+\mu_{2}\right)-p_{c}\left(n+\mu_{1}\right)} \eta_{l_{0}}^{p_{c}}, \quad \omega_{0}<t<\omega_{1},
$$

where

$$
\omega_{1}=\omega_{0}+\frac{M_{5}}{M_{4}}\left(l_{0} / 2\right)^{-\left[p_{c}(n-q-1)-q\left(n+\mu_{2}\right)\right] /\left(p_{c}-q\right)} .
$$

Hence

$$
\begin{aligned}
\eta_{l_{0}}\left(\omega_{1}\right) \geq & \eta_{l_{0}}\left(\omega_{0}\right)+\frac{1}{2} M_{2}^{-p_{c}} l_{0}^{\left(n+\mu_{2}\right)-p_{c}\left(n+\mu_{1}\right)}\left(\Lambda-\sigma_{0}\right)^{p_{c}}\left(\omega_{1}-\omega_{0}\right) \\
\geq & \eta_{l_{0}}\left(\omega_{0}\right)+\frac{1}{2} M_{2}^{-p_{c}} l_{0}^{\left(n+\mu_{2}\right)-p_{c}\left(n+\mu_{1}\right)}\left(\Lambda-\sigma_{0}\right)^{p_{c}} \\
& \cdot \frac{M_{5}}{M_{4}}\left(l_{0} / 2\right)^{-\left[p_{c}(n-q-1)-q\left(n+\mu_{2}\right)\right] /\left(p_{c}-q\right)} .
\end{aligned}
$$

Notice

$$
\left(n+\mu_{2}\right)-p_{c}\left(n+\mu_{1}\right)-\left[p_{c}(n-q-1)-q\left(n+\mu_{2}\right)\right] /\left(p_{c}-q\right)=0,
$$

one gets

$$
\int_{\mathbb{R}^{n}}(|x|+1)^{\mu_{1}} u\left(x, \omega_{1}\right) d x \geq \eta_{l_{0}}\left(\omega_{1}\right) \geq \eta_{l_{0}}\left(\omega_{0}\right)+\delta_{0} \geq \Lambda-\sigma_{0}+\delta_{0},
$$

with a positive constant

$$
\delta_{0}=\frac{M_{2}^{-p_{c}} M_{5}}{2 M_{4}}\left(\Lambda-\sigma_{0}\right)^{p_{c}} 2^{\left[p_{c}(n-q-1)-q\left(n+\mu_{2}\right)\right] /\left(p_{c}-q\right)}
$$

independent of $l_{0}$. Similarly, we reason

$$
\eta_{\left(2 l_{0}\right) / 2}\left(\omega_{1}\right)=\eta_{l_{0}}\left(\omega_{1}\right) \geq \Lambda-\sigma_{0}+\delta_{0} \geq \Lambda-\sigma_{0} .
$$


The same argument yields

$$
\int_{\mathbb{R}^{n}}(|x|+1)^{\mu_{1}} u\left(x, t_{2}\right) d x \geq \eta_{2 l_{0}}\left(\omega_{2}\right) \geq \eta_{2 l_{0}}\left(\omega_{1}\right)+\delta_{0} \geq \Lambda-\sigma_{0}+2 \delta_{0}
$$

with

$$
\omega_{2}=\omega_{1}+\frac{M_{5}}{M_{4}} l_{0}^{-\left[p_{c}(n-q-1)-q\left(n+\mu_{2}\right)\right] /\left(p_{c}-q\right)} .
$$

Repeating the procedure, one can show that

$$
\int_{\mathbb{R}^{n}}(|x|+1)^{\mu_{1}} u\left(x, \omega_{k}\right) d x \geq \eta_{2^{k-1} l_{0}}\left(\omega_{k}\right) \geq \eta_{2^{k-1} l_{0}}\left(\omega_{k-1}\right)+\delta_{0} \geq \Lambda-\sigma_{0}+k \delta_{0}
$$

with

$$
\omega_{k}=\omega_{k-1}+\frac{M_{5}}{M_{4}}\left(2^{k-2} l_{0}\right)^{-\left[p_{c}(n-q-1)-q\left(n+\mu_{2}\right)\right] /\left(p_{c}-q\right)}, \quad k=1,2, \ldots .
$$

Therefore

$$
\sup _{t>0} \int_{\mathbb{R}^{n}}(|x|+1)^{\mu_{1}} u(x, t) d x=+\infty
$$

which contradicts (19).

Now, for the general case without the assumption that $u(x, t)$ is nonincreasing, consider $\underline{u}(x, t)$ defined by (12), which is nonincreasing and satisfies (13) and (14). Therefore, the conclusions of Lemmas 4.1-4.3 are all valid for $\underline{u}$. Similar to the above argument, one can show that $\underline{u}$ blows up in some $0<T_{*}<+\infty$, and thus $u$ is a blow-up solution.

\section{Competing interests}

The authors declare that they have no competing interests.

\section{Authors' contributions}

All the authors contributed to each part of this study equally and approved the final version of the manuscript.

\section{Author details}

${ }^{1}$ School of Mathematics, Jilin University, Changchun, 130012, China. ${ }^{2}$ School of Mathematics and Statistics, Beihua

University, Jilin, 132013, China. ${ }^{3}$ College of Computer Science and Technology, Jilin University, Changchun, 130012, China.

\section{Acknowledgements}

This work is supported by the National Natural Science Foundation of China (Grant Nos. 11222106 and 11571137).

Received: 9 May 2016 Accepted: 29 July 2016 Published online: 09 August 2016

\section{References}

1. Fujita, H: On the blowing up of solutions of the Cauchy problem for $\frac{\partial u}{\partial t}=\Delta u+u^{1+\alpha}$. J. Fac. Sci., Univ. Tokyo, Sect. 1A, Math. 13, 109-124 (1966)

2. Weissler, FB: Existence and non-existence of global solutions for semilinear equation. Isr. J. Math. 6, $29-40$ (1981)

3. Deng, K, Levine, HA: The role of critical exponents in blow-up theorems: the sequel. J. Math. Anal. Appl. 243(1), $85-126(2000)$

4. Levine, HA: The role of critical exponents in blow-up theorems. SIAM Rev. 32(2), 262-288 (1990)

5. Cao, Y, Yin, JX, Wang, CP: Cauchy problems of semilinear pseudo-parabolic equations. J. Differ. Equ. 246(12), 4568-4590 (2009)

6. Guo, W, Wang, X, Zhou, M: Asymptotic behavior of solutions to a class of semilinear parabolic equations. Bound Value Probl. 2016, 68 (2016)

7. $\mathrm{Li}, \mathrm{H}$, Wang, $\mathrm{X}, \mathrm{Nie}, \mathrm{Y}, \mathrm{He}, \mathrm{H}$ : Asymptotic behavior of solutions to a degenerate quasilinear parabolic equation with a gradient term. Electron. J. Differ. Equ. 2015, 298 (2015) 
8. Martynenko, AV: Fujita-type theorems for doubly degenerate parabolic equations with a time-weighted source. Appl. Anal. 95(5), 1050-1058 (2016)

9. Quirós, F, Rossi, JD: Blow-up sets and Fujita type curves for a degenerate parabolic system with nonlinear boundary conditions. Indiana Univ. Math. J. 50, 629-654 (2001)

10. Wang, CP: Asymptotic behavior of solutions to a class of semilinear parabolic equations with boundary degeneracy. Proc. Am. Math. Soc. 141(9), 3125-3140 (2013)

11. Wang, CP, Zheng, SN: Critical Fujita exponents of degenerate and singular parabolic equations. Proc. R. Soc. Edinb., Sect. A 136(2), 415-430 (2006)

12. Wang, CP, Zheng, SN: Fujita-type theorems for a class of nonlinear diffusion equations. Differ. Integral Equ. 26(5-6), 555-570 (2013)

13. Wang, CP, Zheng, SN, Wang, ZJ: Critical Fujita exponents for a class of quasilinear equations with homogeneous Neumann boundary data. Nonlinearity 20, 1343-1359 (2007)

14. Zheng, SN, Song, XF, Jiang, ZX: Critical Fujita exponents for degenerate parabolic equations coupled via nonlinear boundary flux. J. Math. Anal. Appl. 298, 308-324 (2004)

15. Zheng, SN, Wang, CP: Large time behavior of solutions to a class of quasilinear parabolic equations with convection terms. Nonlinearity 21(9), 2179-2200 (2008)

16. Galaktionov, VA: Conditions for nonexistence in the large and localization of solutions of the Cauchy problem for a class of nonlinear parabolic equations. Ž. Vyčisl. Mat. Mat. Fiz. 23(6), 1341-1354 (1983) (in Russian); English translation: USSR Comput. Math. Math. Phys. 23(6), 36-44 (1983)

17. Galaktionov, VA: Blow-up for quasilinear heat equations with critical Fujita's exponents. Proc. R. Soc. Edinb., Sect. A 124(3), 517-525 (1994)

18. Qi, YW, Wang, MX: Critical exponents of quasilinear parabolic equations. J. Math. Anal. Appl. 267(1), 264-280 (2002)

19. Qi, YW: The critical exponents of parabolic equations and blow-up in $R^{n}$. Proc. R. Soc. Edinb., Sect. A 128(1), 123-136 (1998)

20. Wu, Z, Zhao, J, Yin, J, Li, H: Nonlinear Diffusion Equations. World Scientific, River Edge (2001)

\section{Submit your manuscript to a SpringerOpen ${ }^{\ominus}$ journal and benefit from:}

- Convenient online submission

Rigorous peer review

- Immediate publication on acceptance

- Open access: articles freely available online

- High visibility within the field

- Retaining the copyright to your article 